\title{
O OBJETO DA PRODUÇÃO CIENTÍFICA EM ENFERMAGEM NA UNIVERSIDADE FEDERAL DO RIO GRANDE DO NORTE*
}

\author{
Bertha Cruz Enders**
}

\begin{abstract}
RESUMO: Análise da produção científica dos docentes de enfermagem e dos discentes da pós-graduação, da Universidade Federal do Rio Grande do Norte, 1974-1992. Objetivou-se identificar as linhas de pesquisa e seus pesquisadores, na perspectiva da formação de núcleos de pesquisa em enfermagem. A produção científica correspondeu às publicações e/ou apresentações em eventos científico-culturais e monografias, teses ou dissertações. O objeto de 159 trabalhos de docentes e 27 de discentes foram classificados segundo as prioridades de pesquisa do CNPq / ABEn. Encontrouse tendência para três vertentes, consideradas linhas de pesquisa emergentes: recursos humanos em enfermagem, fundamentação da assistência e enfermagem como prática social. Os resultados subsidiam o planejamento do programa de pós-graduação em enfermagem da UFRN.
\end{abstract}

ABSTRACT: Description study of the scientific production of the faculty and specialization students of the Federal University of the State of Rio Grande do Norte from 1974 to 1992. The objective was to organize the knowledge produced and identify the research teams. A total of 159 faculty works and 27 student monographs were classified according to its theme and the CNPq/ABEn research priorities. It was found that there is a strong tendency for research in three areas: human resources in nursing, fundamentals of nursing care, and nursing as a social institution. The results are being used to orient the planning of the graduate program presently being conducted.

\section{INTRODUÇÃO}

É geralmente reconhecido que a produção científica de Enfermagem no Brasil tem sido fruto dos programas de pós-graduação a nível de mestrado e doutorado. ${ }^{(3,8,11)}$ Tal produção surge tanto dos alunos que, por exigência dos Cursos, elaboram dissertações, quanto dos professores que realizam pesquisa em áreas espcíficas, de forma coletiva ou isoldada.

Demonstrando uma preocupação com a avaliação desse saber, vários autores $(1,3,4,11)$ têm realizado análises dentro dos mais diversas perspectivas, sendo que, a maioria desses estudos utilizam como fonte de dados a produção científcia na pós-graduação e os periódicos científicos nacionais.
No entanto, a produção decorrente de instituições de ensino que não oferecem cursos de pós-graduação "stricto sensu", e evidenciada através de artigos publicados e estudos e pesquisas apresentadas em eventos científico-culturais, não tem sido considerada como parte integrante do conhecimento científico na área. Ainda, alguns autores ${ }^{(11)}$ apontam que essa produção, representada em termos estrictamente quantitativos, é um indicador fraco da atividade de pesquisa. Porém, há consenso ${ }^{(2,6)}$ na afirmação de que, a divulgação da produção científica em enfermagem está concentrada nos eventos científico-culturais das associações; e que em enf ermagem, o nível de publicações em periódicos científicos indexados, tanto nacionais como internacional, ainda é muito baixo. Segue então o questionamento se essa produção, considerada de

\footnotetext{
* Trabalho apresentado como tema livre no $45^{\circ}$ Congresso Brasileiro de Enfermagem. Recife-PE 28 de Novembro a 3 de Dezembro de 1993. ,

** Doutora em Enfermagem, Prof essora Adjunta do Departamento de Enf ermagem, Universidade Federal do Rio Grande do Norte.
} 
natureza "técnica ou artística", (6:46) deverá permanecer fora do âmbito do conhecimento da Enfermagem, porque não esta apresentada graficamente.

Ressalta-se aqui a complexidade e diversidade do conhecimento de Enfermagem. CARPER ${ }^{(7)}$, discorre sobre os quatro tipos de conhecimento em Enfermagem: o empírico científico, o estético ou artístico, conhecimento pessoal e o ético, que foram identificados através de uma análise sintética e conceitual da estrutura do conhecimento de enfermagem. Reconhecendo que o conhecimento científico é o mais valorizado pelo fato de ser empírico, fatual e de natureza teórico-explicativa, a autora ressalta a interrelação e interdependência existentes entre eles e a necessidade da inclusão de todas as formas de conhecimento para o desenvolviemnto da enfermagem.

A implementação do Banco de Dados LILACS em Enfermagem, sob a coordenação de BIREME (Biblioteca Regional de Medicina / OPS) na Universidade Federal de Minas Gerais ${ }^{(10)}$, é um avanço no sentido de divulgação desse conhecimento, porque cataloga o conhecimento verificável em publicações aquele que não é discursivo nos modelos de publicação, porém foram apresentados em congressos e eventos culturais de enfermagem.

Este trabalho tem por finalidade caracterizar as tendências na produção científica-cultural do corpo docente e discente do Departamento de Enfermagem da UFRN, desde seu início em 1974 até 1991. Objetiva, especificamente: 1) identificar os objetos de estudo, que possam futuramente, ser consolidados em linhas de pesquisa, e 2) identificar os grupos de pesquisadores envolvidos nas temáticas.

A localização de grupos de pesquisadores em fase de desenvolvimento, é importante, na perspectiva de formação de linhas de pesquisa e de núcleos de estudo, quer para estruturar a produção científica nos programas de pós-graduação, quer para atender as necessidadess de solução de problemas de saúde e de enfermagem. Nesse sentido, ALMEIDA ${ }^{(2)}$ infere que é preciso alcançarmos:

"a organização da pesquisa em linhas com projetos nas quais há a convergência de pesquisadores na formação de grupos... A organização da pesquisa não se dá de forma mecânica e nem externa aos pesquisadores, ela vai se conformando no próprio processo de produção '"(p.46) não podendo, portanto, ser deixado à desordem.
A necessidade dessa análise torna-se evidente tendo em vista o estudo de viabilidade para a implantação de um programa de pós-graduação "lato-sensu" e "stricto sensu", ora em andamento na UFRN.

\section{REFERENCIAL TEÓRICO}

Neste trabalho, parte-se do pressuposto de que a essência dessa produção representa uma contribuição ao avanço científico da enfermagem e que sua análise deverá ser direcionada para a sua organização em áreas específicas e para as questões básicas de saúde que atende. Como refere ANGERAMI ${ }^{(1: 18)}$, "a pesquisa, sendo o ponto de partida e de chegada da práxis deve contribuir para a identificação de problemas e busca de soluções". Segue portanto, que qualquer avaliação dessa produção deverá considerar a aplicabilidade do conhecimento produzido, em termos de sua contribuição ao desenvolvimento de conhecimento da enfermagem.

O conceito de produção científica docente, aqui utilizado, foi derivado de alguns trabalhos de análise do conhecimento de enfermagem ${ }^{(11,6)}$. Assim, neste estudo, a produção científica docente corresponde a todos os trabalhos de dissertação, teses, monografias, pesquisas, relatórios técnicos, trabalhos apresentados em congressos científico-culturais, artigos e pesquisas publicados ou não, que constarem no "Curriculum Vitae" (CV) do professor. Pressupõe-se que o CV esteja atualizado, seja fidedigno e que os títulos dos trabalhos reflitam a temática do trabalho em si. A produção científica discente refere-se às monografias apresentadas pelos alunos dos Cursos de Especialização, na conclusão do Curso.

O conceito de linha de pesquisa aqui adotada refere-se à

"proposta de investigação sobre um ou vários temas correlacionados, contínuo ao longo do tempo, em questões e problemas relativos às necessidades da população, realizada de modo progressivo e em condições para imediato engajamento de novos pesquisadores ${ }^{\prime}(8: 48)$

\section{MÉTODOS E PROCEDIMENTOS}

O levantamento da produção científica dos docentes foi realizado em 1991, como parte de um diagnóstico preliminar da situação de ensino do Departamento de Enfermagem, apresentado no núcleo de Pós-Graduação em Enfermagem do Nordeste.(9) As informações foram atualizadas até o ano 1992. 
Utilizou-se um formulário para a coleta de dados junto aos docentes. Nesse formulário, o docente era solicitado a fornecer as informações sobre a sua produção científcia desde o ano 1974, o qual deveria provir do Curriculum Vitae, especificando, tipo de produção, título do trabalho, evento, local e data de apresentação e/ou dados de publicação. A produção de 5 docentes que se aposentaram no período de 1991-1992 foi avaliada através das informações em arquivo no Departamento de Enfermagem.

As informações foram relacionadas em ordem cronológica, apuradas e posteriormente agnupadas tematicamente. A unidade de análise foi o título do trabalho, onde o objeto focalizado era identificado. Os descritores do objeto indicavam a temática. A base de análise para os enquadramentos temáticos foi a proposta de "Prioridades de Pesquisa" (Anexo 1) apresentada no $2{ }^{\circ}$ Seminário Nacional de Pesquisa em Enfermagem (SENPE) e divulgada pelo CNPq / ABEn. (5)

A coleta de dados das monografias apresentadas nos Cursos de Pós-graduação foi feita através de revisão direta dos trabalhos e dos relatórios dos Cursos de Especialização utilizando a mesma base classificatória.

Os pesquisadores nas áreas foram identificados a partir do agrupamento dos trabalhos por linha prioritária.

\section{RESULTADOS}

A produção de conhecimento da Enfermagem brasileira foi impulsionada pelos cursos de pós-graduação na década de $70^{(1)}$. Da mesma forma, a produção científica dos docentes do Departamento, teve seu início em 1978 com a elaboração das primeiras teses de mestrado realizadas em outras instituições de ensino, embora tenha havido algumas apresentações científicas iniciais antes desse ano. Um total de 23 teses de mestrato e 01 dissertação de doutoramento foram defendidas dentre os docentes no período de 1978 até 1990.

Os trabalhos citados pelos docentes no formulário de levantamento somaram um total de 159 , no qual estão incluídas as teses de mestrado e doutoramento. Em relação aos 44 docentes em exercício no Departamento de Enfermagem em 1992, observou-se em média 3,6 trabalhos por professor num período de 19 anos.

Essa produção é considerada muito limitada considerando qeu a pós-graduação "lato-sensu" tem 6 anos de existência. Outro fato a considerar, é que 23 professores concluíram cursos de pós-graduação "stricto-sensu" através do Programa de Capacitação Docente da UFRN nesse período, e a expectativa é de que ao retorno, os professores iniciem sua produção científica. Contudo, essa deficiência de produção científica em Enfermagem particularmente na região Nordeste, tem sido documentada em outros estudos. ${ }^{(8)}$ ROCHA et al.(11) encontraram uma proporção parecida nos cursos de pós-graduação "strictosensu", com o mínimo de 0,1 e o máximo de 17,0 trabalhos por docente, num período de dois anos.

Quanto ao ensino de pós-graduação no Departamento de Enfermagem da UFRN, o mesmo teve início no ano de 1983, onze anos após o início do curso de graduação, quando o curso de Especialização no Procedimento Científico de Enfermagem foi oferecido pela primeira vez. Porém, a produção de trabalhos científicos a nível de pós-graduação não foi evidenciada até 1986, no Curso de Especialização em Enfermagem Materno-Infantil, quando a monografia científica foi incorporada como requisito de final do curso. A partir daí, todos os cursos de Especialização realizados no Departamento de Enfermagem tiveram monografias apresentadas pelos alunos. No total, 27 (vinte sete) monografias foram apresentadas nos Cursos de Especialização até 1992.

A TABELA 1 mostra a classificação dos trabalhos dos docentes e das monografias dos cursos de Especialização do Departamento, segundo a área e linha de pesquisa.

Como mostra a TABELA 1, a produção cientifica dos docentes se deu de forma diversificada quanto os temas, uma vez que eles se encontram dispersos nas diferentes linhas das tr6es áreas de pesquisa. Porém, se observa uma ligeira concentração nas linhas de "Formação e utilização dos recursos humanos em enfermagem" (25,1\%) e "Fundamentação de assistência, tecnologia e instrumentalização" (21,4\%). Observa-se também, uma outra tendência, embora menos marcante, para as linhas "Enfermagem como prática social" (13,2\%) e "Determinantes do processo saúde e doença" (11,3\%).

Esta diversificação mostra que, embora a produção científica seja limitada, os profesores como grupo, não têm linhas definidas, nas quais concentrem seus esforços de pesquisa. Por outro lado, a ênf ase na área de formação de recursos humanos e na instrumentaçã̃o da assistência, embora não sendo marcantes as quantificações, prometem ser linhas de pesquisa futuras, porque já há docentés trabalhando nas 
Tabela 1 - Produção científica e técnica docente e discente do departamento de enfermagem da UFRN, 1974 a 1992, por área e linha de pesquisa do CNPq/ABEn

\begin{tabular}{|c|c|c|c|c|}
\hline \multirow{2}{*}{ Área e Linha de Pesquisa } & \multicolumn{2}{|c|}{ Docente } & \multicolumn{2}{|c|}{ Discente } \\
\hline & fa & $\%$ & fa & $\%$ \\
\hline \multicolumn{5}{|l|}{ Área 1 - Profissional } \\
\hline Linha 1 - Enfermagem como prática social & 21 & 19,2 & 01 & 04,0 \\
\hline Linha 2 - Formaçăo e utilizaçăo dos recursos humanos em enfermagem & 40 & 25,1 & 01 & 04,0 \\
\hline Linha 3 - Entidades de enfermagem & 07 & 04.5 & - & - \\
\hline \multicolumn{5}{|l|}{ Área 2 - Assistencial } \\
\hline Linha 1 - Fundamentaçăo da assisténcia, tecnologia e instrumentaçăo & 34 & 21,4 & 11 & 40,0 \\
\hline Linha 2 - Autocuidado à saúde & 08 & 05,0 & 05 & 18,0 \\
\hline Linha 3 - Riscos da assisténcia de enfermagem & 02 & 01,2 & - & - \\
\hline Linha 4 - Determinantes do processo saúde e doença & 18 & 11,3 & 08 & 30,0 \\
\hline \multicolumn{5}{|l|}{$\begin{array}{c}\text { Área } 3 \text { - Estrutura, Organização e Funcionamento } \\
\text { das Instituições de Saúde }\end{array}$} \\
\hline Linha 1 - Modelos de assistência de enfermagem & 09 & 05,7 & - & - \\
\hline Linha 2 - Oferta, acessibilidade e utilizaçăo de serviços de saúde & 06 & 03,8 & - & - \\
\hline Linha 3 - Sistema de informaçőes em enfermagem & - & - & - & - \\
\hline Linha 4 - Incorporaçăo de conhecimento à prática profissional & 07 & 04,5 & - & - \\
\hline Linha 5 - Dinămica das organizações & 04 & 02,5 & - & - \\
\hline OUTROS & 03 & 01,8 & - & - \\
\hline TOTAL & 159 & 100,0 & 26 & 100,0 \\
\hline
\end{tabular}

perspectivas desse tema. Cabe agora analisar com maior profundidade essa produção apra detectar seu direcionamento e qualidade, na perspectiva de aglutinar esforços para a formação das linhas.

Na produção dos cursos de Especialização, observa-se uma concentração maior na área assistencial, particularmente nas linhas 1 e 4, "Fundamentação da assist6encia, tecnologia e instrumentação" $(40,0 \%)$ e "Determinantes do processo saúde e doença" $(30,0 \%)$. A linha "Autocuidado à saúde" é notável com $18,0 \%$ das monografias dentro dessa temática.

A partir desses resultados, pode-se afirmar que os trabalhos produzidos nos cursos de Especialização seguem linhas de pesquisa na área assistencial, o que é justificável uma vez que os enfermeiros que procuram o curso de Especialização são, na maioria, enfermeiros de serviços. Porém, ao comparar as linhas mais enfatizadas pelos docentes com as dos discentes na Tabela 1, observa-se uma incompatibilidade entre estes, havendo concordância em apenas uma linha, "Fundamentação da assistência, tecnologia e instrumentação".

Esse resultado confirma o que outros autores têm observado na produção científica da pós-graduação: que a investigação em enfermagem é resultado de projetos isolados e $\mathrm{em}$ algumas vezes desvinculada da área de concentração dos cursos e das linhas de $\operatorname{pesquisa}^{(1)}$.
BARREIRA $^{(6)}$, ao analisar as temáticas dos projetos apresentados ao $\mathrm{CNPq}$ em comparação com os trabalhos apresentados no $6^{\circ}$ Seminário de Pesquisa em Enfermagem (SENPE), também constatou uma grande divergência temática. Enquanto que nos trabalho do SENPE, houve predomínio da área profissional, especificamente na linha "Enfermagem como prática social", o predomínio no CNPq se deu na área assistencial. Houve também, em ambas as fontes, uma concentração de trabalhos na linha "Determinantes do processo saúde-doença".

Com o objetivo de visualizar a dinâmica da produção científica do Departamento de Enfermagem, os trabalhos foram distribuídos em forma de polígono, por ano. (Gráfico 1)

Como se observa no Gráfico 1. houve dois períodos nos quais a produção dos docentes foi acelerada, no ano de 1981 e em 1990. Em 1981, ocorreu a promoção dos professores colaboradores para a função de professor assistente e um dos requerimentos era apresentação de uma monografia científica. Isto suscitou a elaboração de um número significante de monografias pelos docentes pleiteando enquadramento.

Em 1990, o Congresso Brasileiro de Enfermagem promovido pela Associação Brasileira de Enfermagem (ABEn) aconteceu em Natal, $\mathrm{RN}$ e a maioria dos professores do Departamento participou da orga- 
Gráfico 1 - Produção científica dos docentes 1974 a 1992, por ano no departamento de enfermagem, UFRN

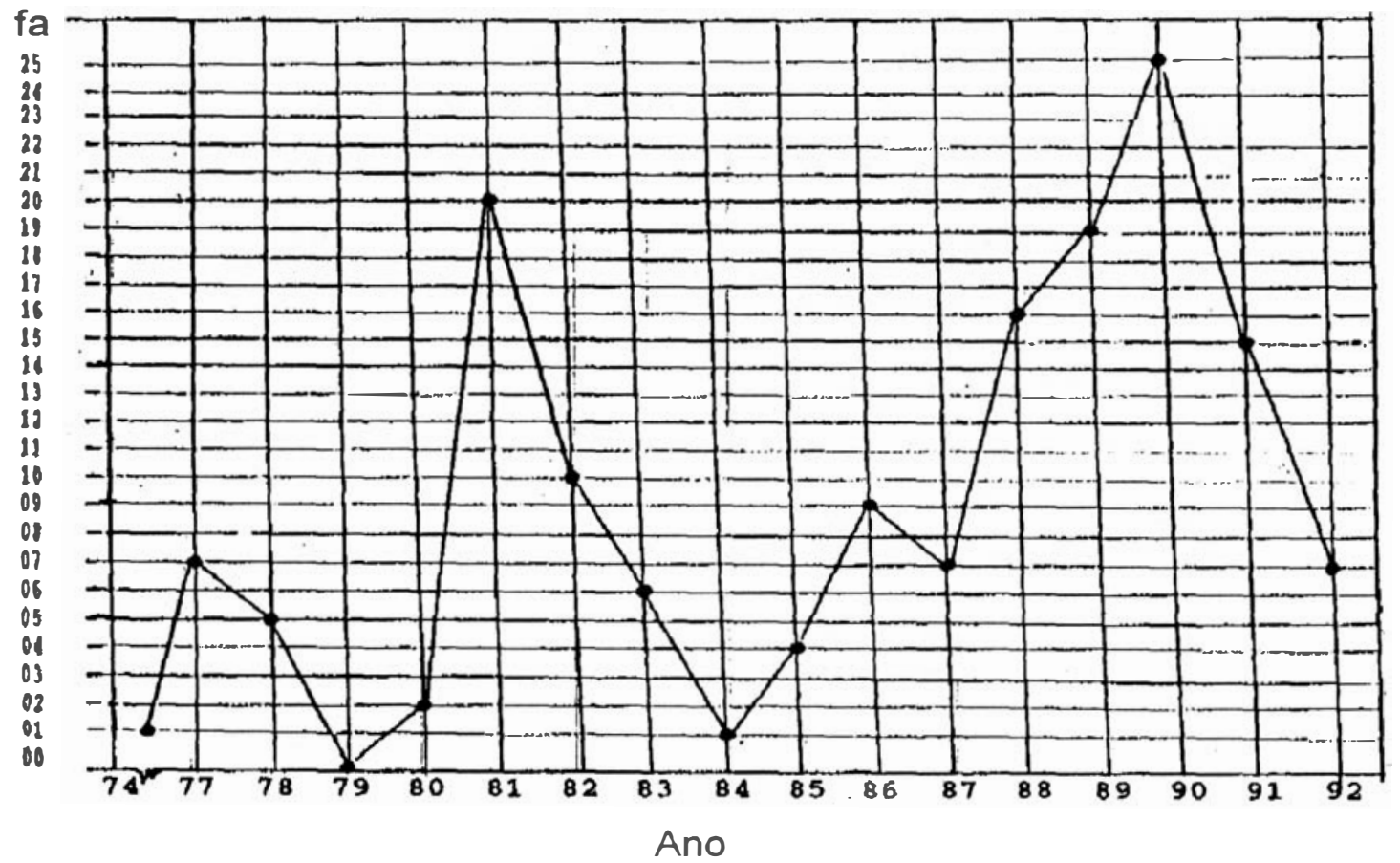

nização do mesmo. Isto representou uma oportunidade para apresentação de trabalhos científicos. Vários ttrabalhos foram apresentados no evento.

Observa-se também na Gráfico 1, que a produção cai marcadamente no ano de 1992, embora esse declínio não chega a ser igual ao ponto mais baixo ocorrido em 1980, que teve apenas 4 trabalhos produzidos.

Espera-se que a produção científica dos docentes continue de forma mais acelerada a partir do ano de 1992, uma vez que o Programa de Pós-Graduação do Departamento está em fase de planejamento, com vistas a um Curso de Enfermagem, a nível de mestrado, a curto prazo.

\section{CONSIDERAÇÕES FINAIS}

Frente aos dados apresentados fazem-se as seguintes afirmações:

1) A produção científica dos docentes do Departamento tem enfatizado a linha de pesquisa que trata da formação dos recursos humanos em enfermagem, indicando assim, uma tendência para os aspectos de ensino, como também para os aspectos profissionais da prática. A esse respeito pode-se afirmar que esse grupo de docentes tra- balhando nessa temática de formação e utilização de recursos humanos representa uma linha de pesquisa emergente, segundo critérios para a avaliação do grau de consolidação utilizados no $\mathrm{CNPq}^{(6)}$. A linha de pesquisa emergente, segundo a autora refere-se ao

"trabalho coletivo realizado em torno de uma questão norteadora, com alguma produção já existente e projetos em andamento, com tend6encias à ampliação $e$ consolidação".'(6:46)

2) A produção científica dos alunos dos Cursos de Especialização concentra-se na área Assistencial. ou scja, no estudo das questões ligadas à prática e do serviço. especificamente na instrumentalização.

3) A produção científica do Departamento de Enfermagem da UFRN tem sido limitada, e há necessidade de uma aceleração continuada e significativa, especialmente frente à proposta vigente no setor de uma ampliação do Programa de Pós-Graduação e à inclusão de um curso "strictosensu".

Para que haja uma coerência interna nos cursos de Pós-Graduação de Enfermagem do Departamento. 
quanto a suas áreas prioritárias e linhas de pesquisa, nas quais os alunos possam se entrosar facilmente, recomenda-se que seja promovida uma discussão mais aprofundada nos interesses de pesquisa do professor. Interessa formar grupos de pesquisa que possamf azer uma programação de pesquisa dentro de sua área de interesse. Dessa forma, os esforços de pesquisa, tão dispendiosos em termos financeiros, energéticos e de recursos humanos, poderão produzir um trabalho mais concentrado e significativo.

Ainda, as atividades de pesquisa de qualquer programa e de pós-graduação, como também seu conteúdo, deverão estar relacionados com a área focalizada no curso e com as necessidades de saúde e da enfermagem da região. Sendo assim, este trabalho constitui uma contribuição para o direcionamento da pesquisa em enfermagem na região e para o planejamento da pós-graduação na área.
É importante ressaltar que o trabalho teve algumas limitações. Em primeiro lugar, os dados foram baseados nos itens do "currículum vitae" de produção científica do professor e providenciados pelo mesmo. Sendo que apenas o título foi analisado, a definição da temática pode ter sido prejudicada, uma vez que o título do trabalho poderá, ou não, representar o objeto real do trabalho. No entanto, acreditase que este impedimento foi mínimo em relação ao total dos trabalhos. Em segundo lugar, alguns trabalhos poderiam ter sido omitidos, uma vez que alguns professores preencheram o formulário sem consulta ao seu "curriculum vitae". E ainda, os professores que se encontravam de licença, ou afastados na época do levantamento, não tiveram oportunidade de atualizar seus currículos para os propósitos do trabalho, e por tanto, poderão ter alguns trabalhos não incluídos nesta análise.

\section{REFERÊNCIAS BIBLIOGRÁFICAS}

1. ALMEIDA, Maria Cecilia Puntel. A pós-graduação e m enf ermagem no Brasil - situação atual. Rev. Lat. Am. Enf.., v. 1 , n. 1, p. 43-50, janeiro 1993.

2. ROCHA, Juan Stuardo Y. O saber de enfermagem

3. e sua dimensão prática. São Paulo: Cortez, 1986. 128p , STEAGALL-GOMES, Daisly L., RUFINO, Márcia C., SILVA, Graciette B. A produção do conhecimento na pós-graduação em Enfermagem no Brasil. In: Congresso Brasileiro de Enfermagem. 33, Manaus, 1981 Anais... Manaus: ABEn, 1981. 174p. p. 110-28.

4. ANGERAMI, Emilia Saporiti. O mister da investigação do enfermeiro. Rev. Lat. Am. Enf., Ribeiro Preto, v. 1, n. 1, p. 11-22, Janeiro 1993

5. BARREIRA, Ieda de Alencar. Informativo: Enfermagem \& CNPq, CNPq., novembro 1991. 11p. (Mimeo.)

6. A pesquisa em enf ermagem no Brasil e sua posição em agência federal de fomento. Rev. Lat. Am. Enf., Ribeirão Preto, v. 1, n. 1, p. 51-57, janeiro 1993.
7. CARPER, Barbara A. Fundamental patterns of knowing in nursing. Advances in Nursing Science, Germantown, Maryland, v. 1.n. 1, p. 12-23, october 1978.

8. CNPq. Avaliação \& Perspectivas, Volume 6, Ciências da saúde. Brasilia: $\mathrm{CNPq}$, Coordenação Editorial, 1983. 38 Enfermagem, p. 135-191.

9. ENDERS, Bertha C. Situação atual e perspectiva do ensino de graduação e pós-graduação no Departamento de Enfermagem de UFRN. Apresentado na I Reunião do Núcleo de Pós-Graduação em Enfermagem do Nordeste, Salvador, Bahia, 2-4 de abril, 1991. (Mimeo)

10. KISIL, Marcos. A Fundação W.K. Kellogg e o desenvolvimento da enfermagem na América Latina. Rev. Lat. Am. Enf., Ribeirão Preto, v. 1, n. 1, p. 37-42, janeiro 1993.

11. ROCHA, Semiramis Melani M., ALMEIDA, M. Cecilia Puntel, WRIGHT, Maria da Glória M., VIEIRA, Terezinha Teixeira. O ensino de pós-graduação. São Paulo: Cortez, 1989. Cademos de Enfermagem, 3. 62p.

Recebido para publicação em 4.12 .93 


\section{Prioridades de pesquisa em enfermagem - CNPq/ABEn (BARREIRA, 1991)}

\section{Área 1 - Profissional}

LINHA 1 - A Enfermagem como prática social

- Objeto de trabalho da enfermagem

- Relação do enfermeiro com sua clientela

- Espaço do enfemeiro no mercado de trabalho

- Fatores culturais, históricos, políticos, sociais, econômicos, determinantes do desenvolvimento profissional.

LINHA 2 - Formação e Utilização de Recursos Humanos em Enfermagem

- Currículos de enfermagem

- Adequação do enfermeiro à prática profissional.

- Integração docente-assistencial.

- Metodologia do ensino-aprendizagem e avaliação desse processo

- Relação mercado de trabalho e enfermagem

- Divisão social do trabalho

- Força de trabalho e recursos humanos (quantitativos e qualitativos)

- Perfis de estudantes dos cursos de enfermagem.

\section{Área 2 - Assistencial}

LINHA 1 - Fundamentação da Assistência, Tecnologia e Instrumentação

- Renovação de procedimentos e metodologias

- Desenvolvimento e testagem de teorias e conceitos de enfermagem

- Adequação de recursos tecnológicos.

LINHA 2 - Autocuidado à Saúde

- Respostas a perguntas sobre condições de ajudar às pessoas

- Difusão do conhecimento sobre saúde.

LINHA 3 - Riscos da Assistência de Enfermagem

- Danos ou prejuízos causados à clientela

LINHA 4 - Determinantes do Processo Saúde-Doença

- Estilo de vida e saúde das populações

- Necessidades e problemas da clintela

- Crenças, atitudes, comportamentos e necessidades em saúde

- Estudos epidemiológicos de morbidade e mortalidade 


\section{Área 3 - Estrutura, Organização e Funcionamento das Instituições de Saúde}

LINHA 1 - Modelos de Assistência de Enfermagem

- Criação e implantação de modelos de assistência

- Custo/eficiência desses modelos

LINHA 2 - Oferta, Acessibilidade e Utilização de Serviços de Saúde

- Oferta, demanda dos serviços de saúde

- Distribuição, acessibilidade, expansão de cobertura e utilização dos serviços de saúde

- Organização de serviços

LINHA 3 - Sistema de Informação em Enfermagem

- Informática e enfermagem

- Sistema de comunicação

- Sistema de divulgação e documentação de enfermagem

LINHA 4 - Incorporação de Conhecimento à Prática Profissional

- Produção e utilização do conhecimento de enfermagem

- Utilização de conhecimentos produzidos na melhoria da prática profissional

LINHA 5 - Dinâmica das Organizações

- Organização em movimento

- Conflitos

- Lideranças

- Trabalhos em equipe multi-profissional

Fonte: BARREIRA, leda de Alencar. Informativo: Enfermagem \& CNPq. CNPq, novembro, 1991. 11p. (Mimeo) 